\title{
Functionally Dissociating Aspects of Event Memory: the Effects of Combined Perirhinal and Postrhinal Cortex Lesions on Object and Place Memory in the Rat
}

\author{
Timothy J. Bussey, Janice L. Muir, and John P. Aggleton \\ School of Psychology, Cardiff University, Cardiff CF1 3YG Wales, United Kingdom
}

Reciprocal interactions between the hippocampus and the perirhinal and parahippocampal cortices form core components of a proposed temporal lobe memory system. For this reason, the involvement of the hippocampus in event memory is thought to depend on its connections with these cortical areas. Contrary to these predictions, we found that NMDAinduced lesions of the putative rat homologs of these cortical areas (perirhinal plus postrhinal cortices) did not impair performance on two allocentric spatial tasks highly sensitive to hippocampal dysfunction. Remarkably, for one of the tasks there was evidence of a facilitation of performance. The same cortical lesions did, however, disrupt spontaneous object recognition

Processing of a complex event memory likely involves the association of information from multiple brain regions. One such contributing region may be the perirhinal-parahippocampal cortex (Brown, 1990; Squire and Zola-Morgan, 1991; Eichenbaum et al., 1994; Gaffan and Parker, 1996), which has dense, reciprocal connections with the hippocampus (Deacon et al., 1983; Suzuki and Amaral, 1994). Indeed, it has been proposed that the involvement of the hippocampus in event memory depends on its connections with this cortical area (Squire and Zola-Morgan, 1991; Eichenbaum et al., 1994). A direct prediction is that perirhinalparahippocampal cortex damage should lead to hippocampal dysfunction and that it should not be possible to obtain functional double dissociations between these two regions.

Contrary to these predictions, some studies have revealed dissociations between fornix and perirhinal cortex lesions (Gaffan, 1994a; Ennaceur et al., 1996). This is noteworthy, because fornix lesions partially disconnect the hippocampus and mimic many of the effects of hippocampectomy. In such studies, lesions of perirhinal or perirhinal plus postrhinal cortex (thought to be the homolog of parahippocampal cortex in the monkey; Burwell et al., 1995) impaired spontaneous object recognition but spared certain tests of spatial memory (Ennaceur et al., 1996; Aggleton et al., 1997; Ennaceur and Aggleton, 1997). The opposite pattern of results was obtained after fornix lesions (Ennaceur et al., 1996, 1997). These findings raise the question of whether the visual information required by the hippocampus in spatial memory tasks

Received Sept. 14, 1998; revised Oct. 19, 1998; accepted Oct. 21, 1998.

This research was supported by a project grant from the Wellcome Trust. We thank Alison Baird, Angie Morgan, and Charlotte Price for invaluable assistance with behavioral testing and histology.

Correspondence should be addressed to Dr. Timothy J. Bussey, Laboratory of Neuropsychology, National Institute of Mental Health, National Institutes of Health, Building 49 Room 1B80, Bethesda, MD 20892.

Copyright (C) 1998 Society for Neuroscience $\quad 0270-6474 / 98 / 190495-08 \$ 05.00 / 0$ and object discrimination reversal learning but spared initial acquisition of the discrimination. This pattern of results reveals important dissociations between different aspects of memory within the temporal lobe. Furthermore, it shows that the perirhinal-postrhinal cortex is not a necessary route for spatial information reaching the hippocampus and that object familiaritynovelty detection depends on different neural substrates than do other aspects of event memory.

Key words: episodic memory; event memory; spatial memory; object recognition; perirhinal cortex; postrhinal cortex; temporal lobe; rat must necessarily come from the perirhinal and postrhinal cortices. Resolving this issue is important for understanding the neurobiology of event memory; although spatial memory is not directly equivalent to event memory, it is an important attribute that is likely to depend on hippocampal function (Gaffan, 1994b). The foregoing results thus suggest important dissociations between aspects of event memory, namely of visual object processing in temporal cortex and spatial processing in the hippocampal system (O'Keefe and Nadel, 1978). To test this possibility, however, it is vital to use spatial tasks that unambiguously test allocentric spatial memory, because it is this form of spatial processing that is thought to depend critically on the hippocampus.

Accordingly, rats with excitotoxic lesions of the perirhinalpostrhinal (PPRH) cortex were tested with two well characterized tests of allocentric spatial memory using the Morris water maze and the radial arm maze. To confirm the functional efficacy of the lesions, the same animals were tested on a spontaneous object recognition task. Finally, to extend our investigations into the involvement of these areas in visual processing, the rats were also tested on rewarded one-pair object discriminations and reversal.

\section{MATERIALS AND METHODS}

\section{Surgical and histological methods}

Fifteen adult male rats (DA strain; Bantin-Kingman, Hull, UK) received bilateral injections of NMDA in five sites in the PPRH cortex. This PPRH group was compared with 13 control (CONT) rats that received sham surgeries. All animals were deeply anesthetized by intraperitoneal injection $(60 \mathrm{mg} / \mathrm{kg})$ of pentobarbitone sodium (Sagatal, Rhône Mérieux) and then placed in a stereotaxic head holder (David Kopf Instruments, Tujunga, CA) with the nose bar at +5.0. The scalp was then cut and retracted to expose the skull. For the PPRH lesion, injections of $0.2 \mu \mathrm{l}$ of $0.09 \mathrm{M}$ NMDA (Sigma, Poole, UK) dissolved in phosphate buffer, $\mathrm{pH}$ 7.2, were made through a $1 \mu \mathrm{l}$ Hamilton syringe into five sites in each hemisphere. Each injection was made gradually over a $5 \mathrm{~min}$ period, and the needle was left in situ for an additional 4 min before being withdrawn. The stereotaxic coordinates relative to ear-bar zero were as 
follows: anteroposterior (AP) +3.9, lateral (L) \pm 5.9 , dorsoventral (DV) $+2.0 ; \mathrm{AP}+2.4, \mathrm{~L} \pm 6.1, \mathrm{DV}+1.6 ; \mathrm{AP}+0.6, \mathrm{~L} \pm 6.2, \mathrm{DV}+2.5 ; \mathrm{AP}-0.8$, $\mathrm{L} \pm 6.2, \mathrm{DV}+2.7$; and $\mathrm{AP}-0.8, \mathrm{~L} \pm 6.2, \mathrm{DV}+4.3$. The CONT animals received exactly the same initial surgery, i.e., craniotomy, but no injections were made. At the completion of all surgeries, the skin was sutured, and an antibiotic powder (Acramide; Dales Pharmaceuticals, Skipton, UK) was applied.

On completion of the experiment, all animals were killed with an overdose of Euthatal (Rhône Mérieux) and perfused intracardially with saline, followed by $10 \%$ formol saline. The brains were then removed and placed in $10 \%$ formol saline for a minimum of $2 \mathrm{hr}$. After fixation, the brain was transferred to $20 \%$ sucrose in $0.2 \mathrm{M}$ phosphate buffer and left overnight. The brain was then cut on a freezing microtome into $60 \mu \mathrm{m}$ coronal sections, and sections were mounted and then stained with cresyl violet, a Nissl stain.

\section{Behavioral methods}

Testing began $\sim 30 \mathrm{~d}$ after surgery. For some of the postoperative test period (radial arm maze task; see below), the animals were placed on a restricted diet, but they were weighed regularly and their food was adjusted to ensure that they did not fall below $85 \%$ of normal body weight. Throughout the study, all animals had access to water ad libitum. All animals were tested on the various tasks in the order in which they are described below.

Morris swim task. Eight equidistant start locations [north $(\mathrm{N})$, south (S), east (E), west (W), NE, SE, NW, and SW] were allocated, thus delineating four quadrants (NE, SE, NW, SW) of the pool. In each trial, a rat was placed in the pool facing the wall at one of the eight start locations. To escape the cool $\left(25^{\circ} \mathrm{C}\right)$ water, the rat swam to an invisible platform, which was positioned in the same place in the same quadrant throughout the acquisition sessions. The position of the platform and the starting positions for each trial were counterbalanced between rats. Animals (PPRH, $n=15$; CONT, $n=13$ ) received four trials per session, one session per day, for ten d. Each acquisition trial was terminated either when the animal located the hidden escape platform or after 120 sec had elapsed. If the rat located the platform, it was allowed to remain there for $30 \mathrm{sec}$. If the rat failed to find the platform after $120 \mathrm{sec}$, it was placed on the platform and allowed to remain on it for $60 \mathrm{sec}$. In the next trial, the rat was placed in the pool at the second start location, and so on for four trials. On the eleventh day, a probe trial was given in which the platform was removed from the pool. Each rat was placed at a start position opposite to where the platform had been located.

Radial arm maze task. The rats were tested next in a standard eightarm radial maze in which two reward pellets were placed at the end of each arm at the start of each test session (one session per day). Normal rats learn the most effective strategy, which is to enter each arm only once. The requirement to update the memory of those arms already entered ensures that the task taxes spatial working memory. Testing was performed in an eight-arm radial maze made of wood. This apparatus was of a standard design, with the exception that the arms had walls made of clear Perspex to ensure that the rats could not cross directly from one arm to another. Each arm was $87 \mathrm{~cm}$ long and $10 \mathrm{~cm}$ wide; the side walls were $24 \mathrm{~cm}$ high. At the end of each arm was a food well $2 \mathrm{~cm}$ in diameter and $0.5 \mathrm{~cm}$ deep. Each arm led through a clear Perspex guillotine door, which was $14 \mathrm{~cm}$ high to the central octagonal arena and $34 \mathrm{~cm}$ in diameter. Each of these guillotine doors had strings attached, enabling the experimenter to open the doors either individually or simultaneously. The arms of the maze were mounted on a turntable so that they stood 62 $\mathrm{cm}$ off the ground. The turntable enabled the arms, but not the central platform, to be rotated through $360^{\circ}$. The floor of the testing room was marked so that the position of the maze could be standardized in relation to the room cues. Lighting was provided by two fluorescent lights mounted $180 \mathrm{~cm}$ above the maze.

After habituation to the maze, training consisted of 12 trials, one per day. (One PPRH and three CONT animals would not explore the maze; these animals were therefore excluded from this phase of the study. The numbers of subjects in each group for this phase of the study were therefore PPRH, $n=14$ and CONT, $n=10$.) At the start of each trial, all eight arms were baited with two $45 \mathrm{mg}$ reward pellets (Sandown Instruments). The animal was placed in the central arena and was allowed to explore the maze and collect pellets until all eight arms had been visited. The number of arm entries taken to retrieve all pellets and the number of correct choices (visits to baited arms) were recorded. After the 12 acquisition days, rats were given five trials, one per day, designed to control for the possible use of nonallocentric strategies. The rat was placed in the maze as usual and allowed to collect pellets from any four arms. It was then removed from the apparatus and placed in a carrying cage for $30 \mathrm{~min}$. During this period, the maze was rotated $45^{\circ}$ either clockwise or counterclockwise, and the four unvisited positions were baited. Now the unvisited baited arms were in the same location relative to the room cues as they would have been had the maze not been rotated but the actual arms had changed. After the delay, the rat was placed back in the central arena and was allowed to revisit the four remaining baited arms. The experimenter recorded the number of errors (entries into unbaited arms) made after the delay.

Spontaneous object recognition test. The apparatus consisted of an open arena $(100 \times 100 \times 46 \mathrm{~cm})$ made of wood, the inside of which was painted gray. The floor was covered with sawdust. The arena was situated in a room containing features such as a door, light fixtures, and a video camera. Triplicate copies were obtained of the objects to be discriminated, which were made of glass, plastic, or metal. For any given test, the pairs of objects to be discriminated were typically composed of the same material so that they could not readily be distinguished by olfactory cues. All rats (PPRH, $n=15$; CONT, $n=13$ ) received a series of habituation sessions before the first test.

Each test session consisted of two phases. In the initial sample phase, two identical objects (A1 and A2) were placed in the far corners of the arena, each $10 \mathrm{~cm}$ from the side wall. A rat was then placed in the middle of the arena, and the total time spent exploring the two objects was determined from video-taped recordings. Exploration of an object was defined as directing the nose to the object at a distance of $<2 \mathrm{~cm}$ and/or touching it with the nose. This "sample phase" ended when the rat had explored the two identical objects for a total of $25 \mathrm{sec}$. The rats' behavior was assessed from video recordings, and all assessments were blind.

After a delay of $15 \mathrm{~min}$, the rat was reintroduced to the arena ("choice phase"), which now contained a third identical copy of the familiar object (A3) and a new object (B). These were placed in the same locations as the sample stimuli. The location of the two choice objects was counterbalanced between rats and across sessions. All rats were tested with two sets of objects and received a total of four tests. Thus, in test 1, object A was the sample and object B was the novel alternative. For test 2 (48 hr later), their roles were reversed, i.e., object B was the sample and object A was the "novel" alternative. For tests 3 and 4, new pairs of objects were used (C and D) in a similar counterbalanced order. The time spent exploring the novel and familiar objects was recorded for the all $3 \mathrm{~min}$ of the choice session, but attention was focused on the first minute, during which object discrimination is typically greatest (Dix and Aggleton, 1998). From these results, we calculated d1, the difference in time spent exploring the novel and familiar objects for each of the two sets of objects, and d2, the proportion of total exploration time spent exploring the novel objects for each pair of objects (i.e., d1 divided by the total time spent exploring the objects). This latter measure takes into account individual differences in the total amount of exploration time.

Object discriminations and reversal. The apparatus, a Grice box, consisted of a small rectangular start box $(13 \times 18 \mathrm{~cm})$ separated from a triangular test area by a guillotine door. The far wall of the test area was $43 \mathrm{~cm}$ long and $43 \mathrm{~cm}$ from the guillotine door. The walls of the apparatus, which were made of aluminum, were $24 \mathrm{~cm}$ high. The floor contained two food wells, $2.5 \mathrm{~cm}$ in diameter, positioned $35 \mathrm{~cm}$ from the start box. An aluminum partition, which protruded $16 \mathrm{~cm}$ from the far wall, ensured that the rats could not run directly between the two food wells, which were $21 \mathrm{~cm}$ apart. During pretraining, the rats were trained to run from the start box to find food pellets in either food well by pushing aside a circular wooden disk ( $4.5 \mathrm{~cm}$ diameter, $1.5 \mathrm{~cm}$ high). This was followed by two object discriminations. The objects were comparable to those used in the object recognition task. For half of the animals in a group, one of the two objects $(\mathrm{S}+)$ was rewarded with two food pellets, and for the other half, the other object was rewarded. For each trial, the guillotine door was raised, and the rat were allowed to select one object. A choice occurred when the rat had displaced the object sufficiently to reveal the edge of the food well. The left-right positions of the objects were varied according to a pseudorandom schedule, and there was no correction procedure.

All rats (PPRH, $n=15$; CONT, $n=13$ ) were tested on the first object discrimination for $6 \mathrm{~d}$, followed by the second object discrimination for $4 \mathrm{~d}$. Each session consisted of 20 trials. Next, the rats were given a 1 week retention period, after which the second object discrimination was re- 

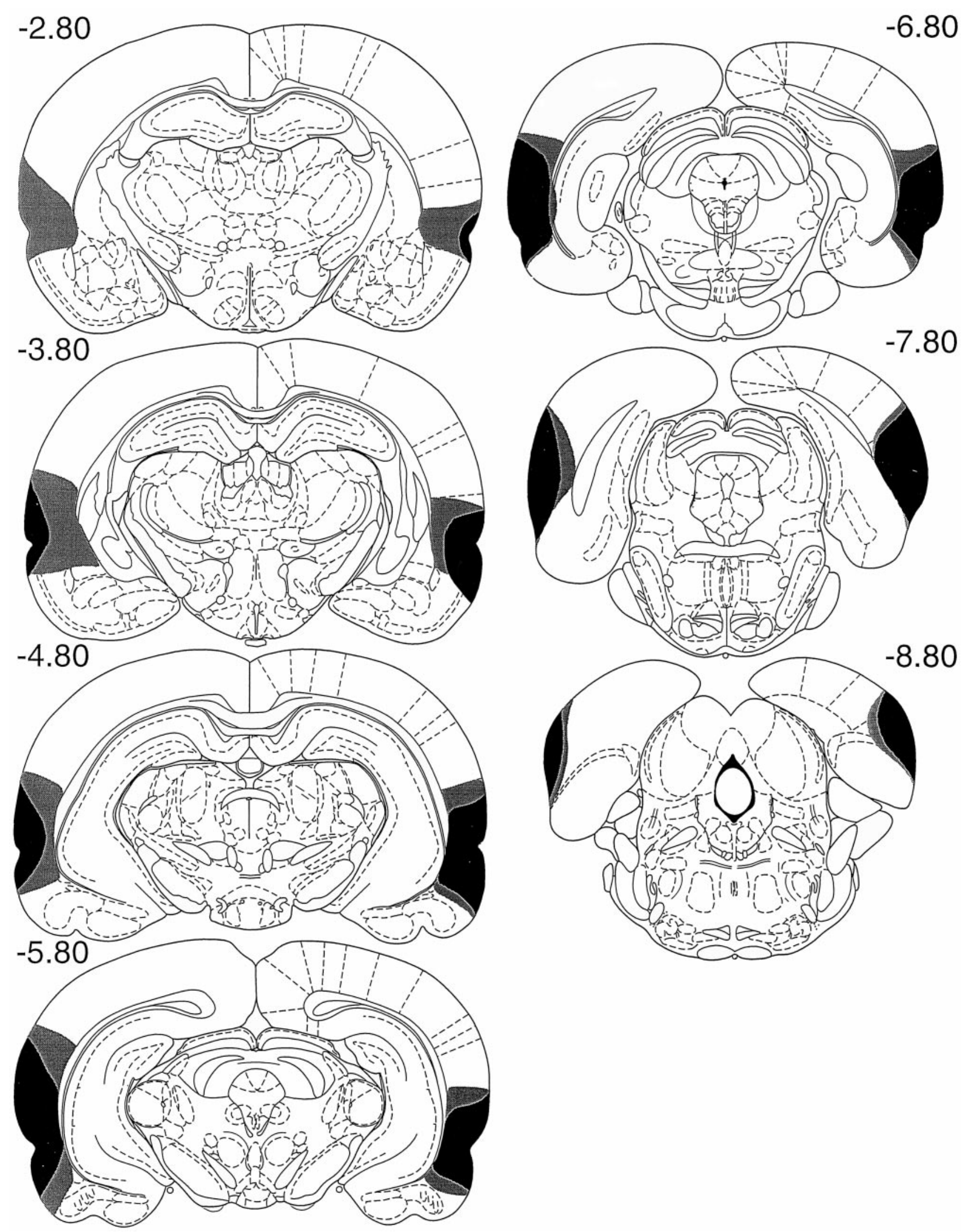

Figure 1. Coronal sections illustrating the extent of the largest ( gray) and smallest (black) lesions of the PPRH cortex. The numbers correspond to the approximate position from bregma (Paxinos and Watson, 1997). 

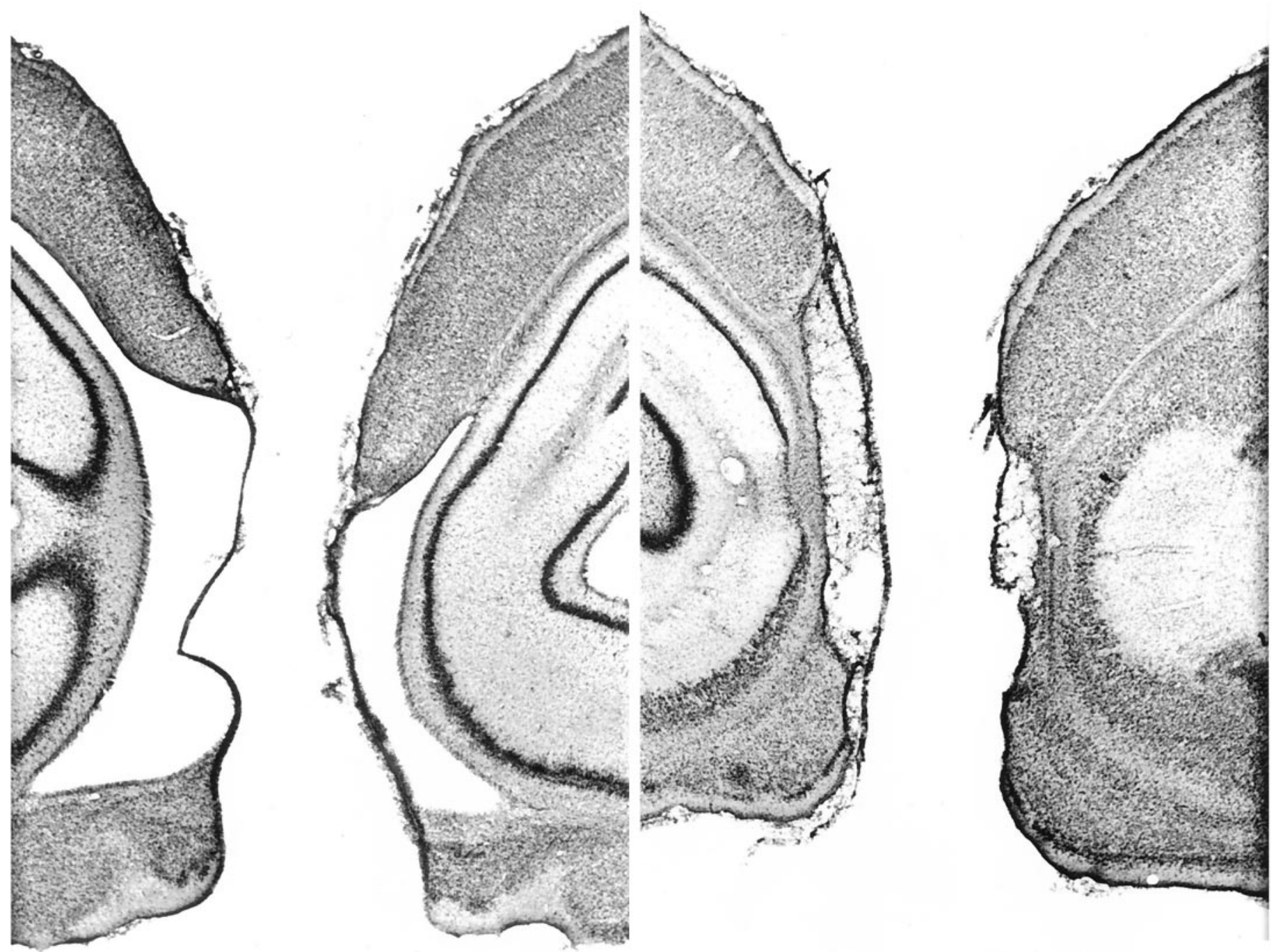

Figure 2. Photomicrographs showing the extent of a typical PPRH lesion. Left, The lesion in the left and right hemispheres at approximately bregma -4.0 and -4.8 (Paxinos and Watson, 1997). Right, The lesion in the left and right hemispheres at approximately bregma -6.8 and -8.0 (Paxinos and Watson, 1997). Note the very small amount of CA1 damage in the left section in the right panel. This is a typical amount of CA1 damage in cases in which hippocampal damage occurred.

tested for one session, followed by a 2 week retention period, after which this same discrimination was retested for three sessions. (One PPRH animal suddenly became ill during this test and was therefore excluded from the remainder of the study; the numbers of subjects in each group for this phase of the study were therefore PPRH, $n=14$ and CONT, $n=$ 13.) Finally, animals were tested on a reversal of this discrimination in which reward contingencies were reversed (i.e., the $\mathrm{S}+$ became the $\mathrm{S}-$ and vice versa). (One PPRH animal would not work under the reversal condition and was therefore excluded; the numbers of subjects in each group for this phase of the study were therefore PPRH, $n=13$ and CONT, $n=13$ ). Reversal testing continued for 12 sessions, one session per day. All data from discriminations and reversal were analyzed across sessions with two-way ANOVA. Reversal data were further analyzed in terms of errors required to progress between three learning stages: "perseveration" ( $<6$ of 20 correct, during which animals are responding to the previously rewarded stimulus); "chance performance" (6-13 of 20 ); and "new learning" ( $>14$ of 20 , during which animals are responding to the currently rewarded stimulus) (for review, see Jones and Mishkin, 1972; Dias et al., 1996; Bussey et al., 1997).

Activity. After completion of the above tests, all rats (PPRH, $n=13$; CONT, $n=13)$ were placed in novel test cages $(56 \times 39 \times 19 \mathrm{~cm})$ in a novel room. Activity was measured using pairs of photobeams situated 20 $\mathrm{cm}$ apart and $18 \mathrm{~cm}$ from the end of the cage (Paul Fray Ltd., Cambridge,
UK). The total number of beam breaks was recorded. Data were gathered in 12 intervals of 10 min each.

\section{RESULTS}

\section{Histological results}

Histological analysis showed that PPRH animals had very extensive cellular loss throughout the perirhinal and postrhinal cortices, as well as area TE (Figs. 1, 2). The lesions started close to the rostral border of the perirhinal cortex and continued caudally throughout the postrhinal cortex. Within this region, all neurons had disappeared. In two animals, there was some unilateral sparing of the most rostral perirhinal cortex. The lesions consistently extended ventrally to include adjacent parts of the pyriform cortex and lateral entorhinal cortex. The cellular damage also extended dorsally so that in 13 cases it reached the ventral border of the primary auditory cortex. In eight cases, there was unilateral cell loss in a very restricted portion of the hippocampal CA1 field, and in a further three cases, this damage was bilateral. 
a

\author{
Morris Swim Task \\ Acquisition
}

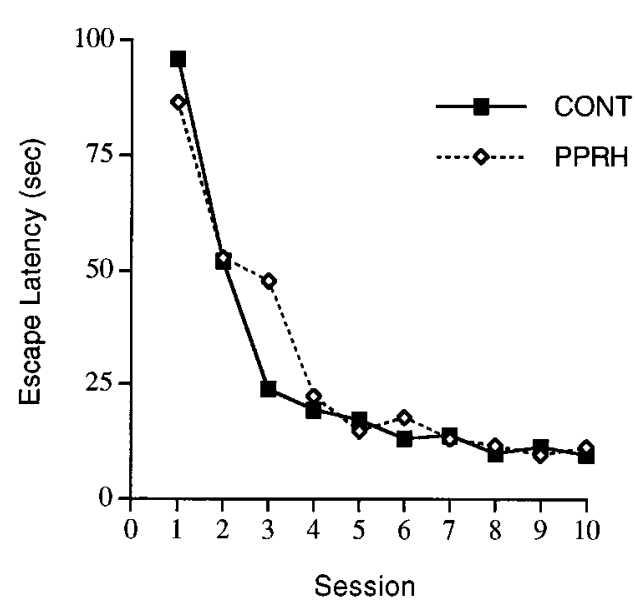

b
Morris Swim Task
Acquisition

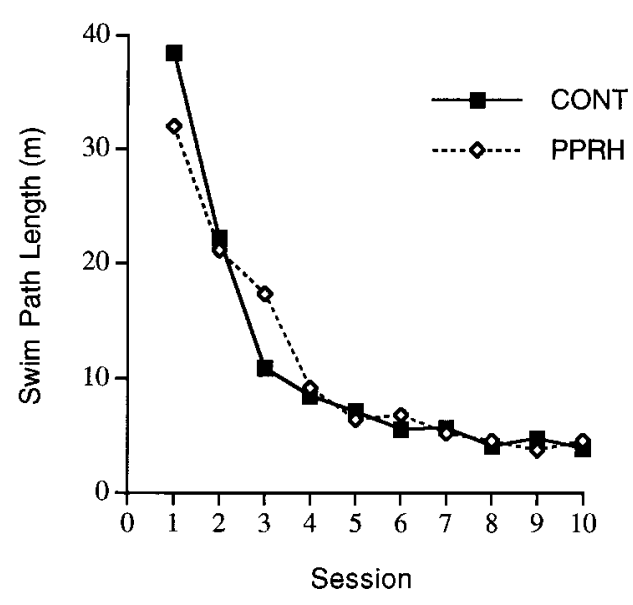

C
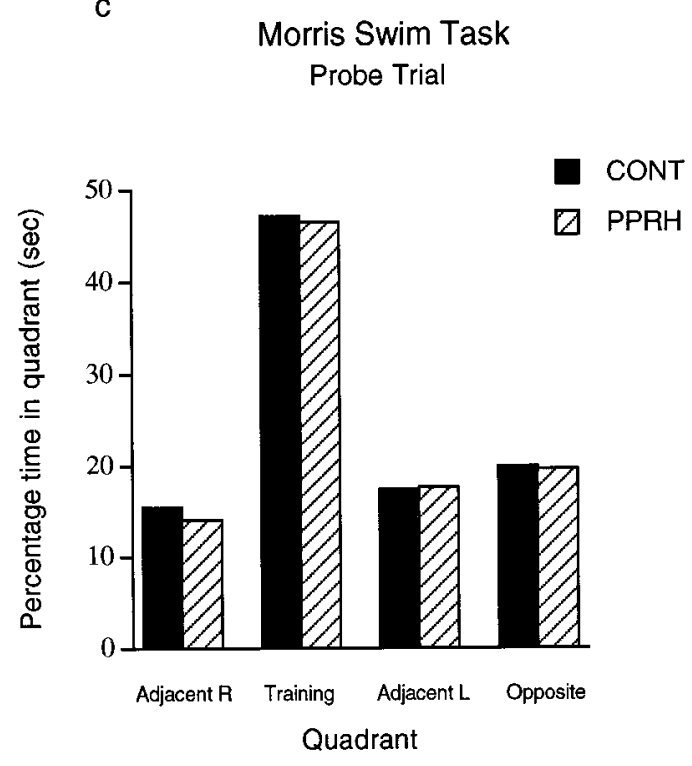

\section{Behavioral results}

\section{Morris water maze}

Performance, as measured by latency to reach the platform, showed that the PPRH and CONT animals acquired the task at very similar rates (main effect of group, $F<1$; group $\times$ session interaction, $F_{(9,234)}=1.2$ ) (Fig. $\left.3 a\right)$. Analysis of swim path lengths similarly did not reveal any group differences (main effect of group, $F<1$; group $\times$ session interaction, $F<1$ ) (Fig. $3 b$ ). A probe trial in session 11 in which the hidden platform was removed showed that both groups favored the quadrant where the platform had been and that there was no group difference $(F<1)$ (Fig. 3c).

\section{Radial arm maze}

Both groups rapidly acquired the radial maze task over 12 sessions (main effect of group: number of arms visited, $F_{(1,22)}=2.2$; number correct in first eight choices, $F_{(1,22)}=2.9$ ) (Fig. $\left.4 a, b\right)$, but paradoxically the PPRH animals showed the better level of performance on some accuracy measures [group $\times$ trial interaction: number correct in first eight choices, $F_{(11,242)}=1.98 ; p<0.05$; analysis of simple effects revealed superior performance of PPRH group in trials $3(p<0.05), 11(p<0.001)$, and $12(p<0.05)]$ (Fig. $4 b$ ). Acquisition was immediately followed by five sessions in which each rat was removed from the maze as soon as the first four arms had been selected. During a retention interval of 30 min, the maze was rotated $45^{\circ}$ (clockwise or counterclockwise), and the arms were rebaited so that the unvisited arms were still in the same spatial position with respect to the room cues. The rat was then placed back in the central arena and monitored until all four baited arms had been visited. Both PPRH and CONT animals performed accurately after the delay $(F<1)$ (Fig. 4c), showing that both groups relied on allocentric cues to solve the task.

\section{Spontaneous object recognition}

The effectiveness of the cortical lesion was confirmed with a spontaneous test of object recognition. Normal rats demonstrate object recognition by showing a spontaneous preference for a novel object versus a familiar one. Unlike the CONT group, the PPRH rats showed abnormally low preference levels for the novel object, as measured by the difference in time spent exploring the novel and familiar objects $\left(\mathrm{d} 1, F_{(1,26)}=10.1 ; p=0.004\right)($ Fig. $5 a)$ and by the ratio of total exploration time spent with the novel object $\left(\mathrm{d} 2, F_{(1,26)}=8.1 ; p=0.008\right)$ (Fig. $\left.5 b\right)$.

\section{Object discrimination and reversal}

Despite the recognition deficit, the PPRH rats were able to perform a pair of object discriminations, confirming their ability to distinguish between objects. Both the PPRH and CONT rats rapidly learned to select the rewarded object (main effect of group, $F_{(1,26)}=3.0$; group $\times$ session interaction, $\left.F_{(5,130)}=1.2\right)$. The first discrimination was immediately followed by a second, with a new pair of objects. Again, there were no group differences (main effect of group, $F_{(1,26)}=2.1$; group $\times$ session interaction, $F<1$ ). After the second object discrimination, a 1 week retention interval was interposed, and the rats were retested. There were no

$\leftarrow$

Figure 3. Performance of PPRH and CONT animals on the Morris swim task. $a$, Mean escape latencies during acquisition. $b$, Mean swim path lengths during acquisition. $c$, Percentage of time spent in the four quadrants during a probe test conducted after the tenth acquisition session. There were no differences between the groups during either test. 
a

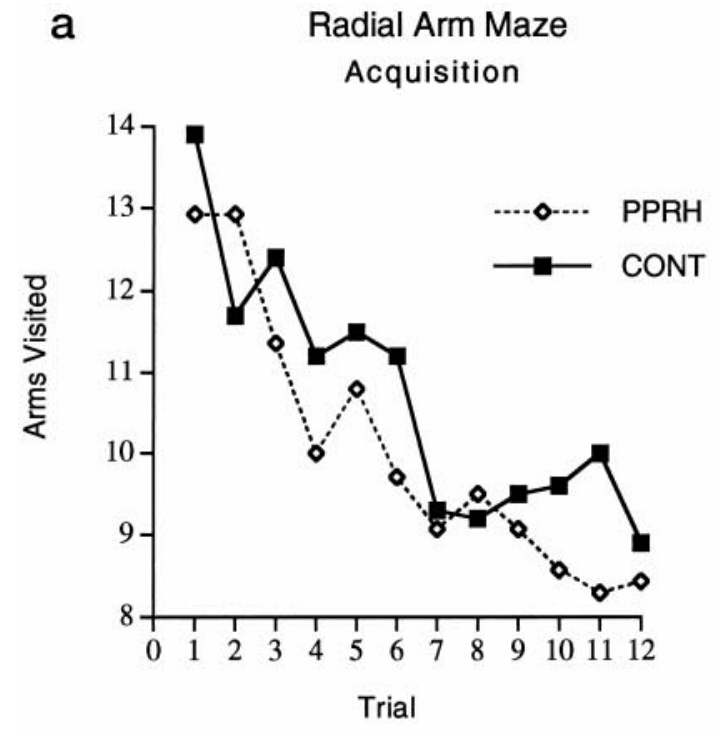

Radial Arm Maze
Acquisition

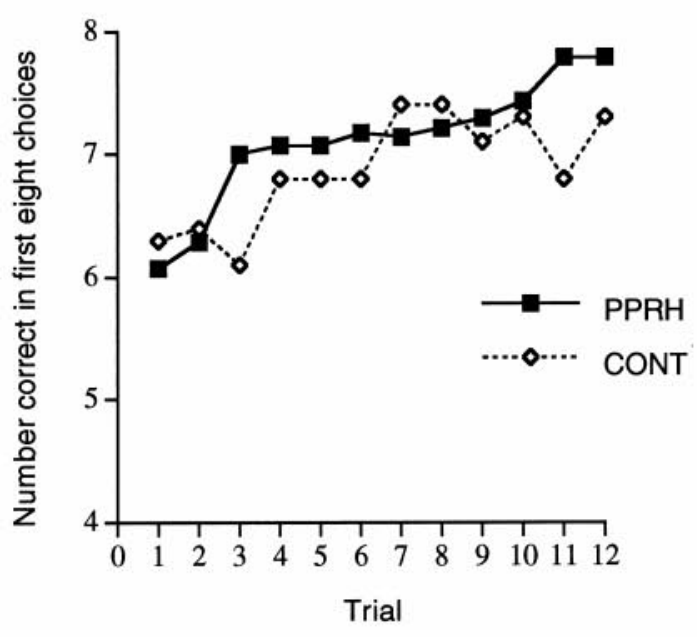

C

$$
\begin{aligned}
& \text { Radial Arm Maze } \\
& \text { Rotation and Delay }
\end{aligned}
$$

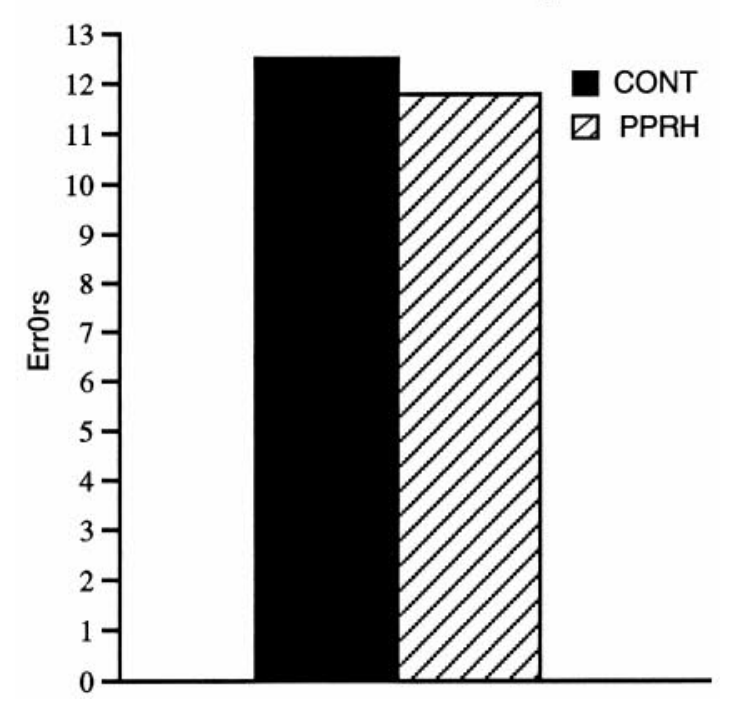

group differences in performance after the 1 week interval $\left(F_{(1,26)}\right.$ $=1.5$ ). After a subsequent 2 week interval, rats were retested over a 3 d period, and PPRH animals were significantly impaired (main effect of group, $F_{(1,25)}=5.1 ; p<0.05$ ), but analysis of simple effects revealed that by the third day of testing PPRH animals had attained the same performance level as controls. Finally, when the reward contingencies of this second discrimination were reversed, the previously rewarded object now being nonrewarded and vice versa, PPRH animals were significantly impaired relative to controls (main effect of group, $F_{(1,24)}=5.8$; $p=0.02$; group $\times$ session interaction $\left.F_{(11,264)}=3.3 ; p<0.001\right)$. Reversal data were further analyzed in terms of errors required to progress between three criteria delineating three learning stages: perseveration, chance performance, and new learning (Jones and Mishkin, 1972; Dias et al., 1996; Bussey et al., 1997). The lack of a significant group $\times$ stage interaction $(F<1)$ suggests that errors were distributed approximately evenly across the three stages. However, PPRH rats tended to make more errors during the perseverative and chance performance stages than did controls.

\section{Activity}

Finally, the rats were given one $2 \mathrm{hr}$ session in novel activity cages fitted with photocells to measure spontaneous locomotor activity. Data were analyzed in 12 intervals of $10 \mathrm{~min}$ each. The spontaneous activity and habituation to the cages was very similar for the PPRH and CONT groups (beam breaks during the first 10 min bin: PPRH, 151.13; CONT, 154.3; beam breaks during final 10 min bin: PPRH, 30.6; CONT, 24.5; main effect of group, $F<$ 1 ; group $\times$ interval interaction, $F<1$ ). Thus, the selective effects of the lesion reported above are unlikely to be attributable to gross changes in arousal or motoric function.

\section{DISCUSSION}

This study provides the first comprehensive test of combined PPRH cortex removal on allocentric spatial memory. The striking finding is that this lesion can impair object recognition yet completely spare spatial memory. Furthermore, the sparing of spatial memory was independent of whether the task taxed spatial "working" or spatial "reference" memory. These results are contrary to several influential theories of event memory that emphasize obligatory interactions between the hippocampus and the perirhinal and parahippocampal cortices (Squire and Zola-Morgan, 1991; Eichenbaum et al., 1994). Instead, they demonstrate a degree of independence between different aspects of memory within the temporal lobe. This feature needs to be accommodated in future models of a temporal lobe memory system.

Although spatial memory is not directly equivalent to event memory, it is an important attribute that is likely to depend on hippocampal function. Recall of an event includes the context or setting in which the event took place, and this contextual information can have a spatial component that is dependent on the hippocampus (Nadel and Willner, 1980; Gaffan, 1994b). Object recognition, in contrast, does not appear to depend on the hip-

$\leftarrow$

Figure 4. Performance of PPRH and CONT animals on the radial arm maze task. $a$, Mean number of arms visited to obtain reward from all eight arms. $b$, Number correct in the first eight choices. $c$, Total number of errors committed across five sessions in which the maze was rotated during a 30 min delay period. 
a

\section{Object Recognition}

d1

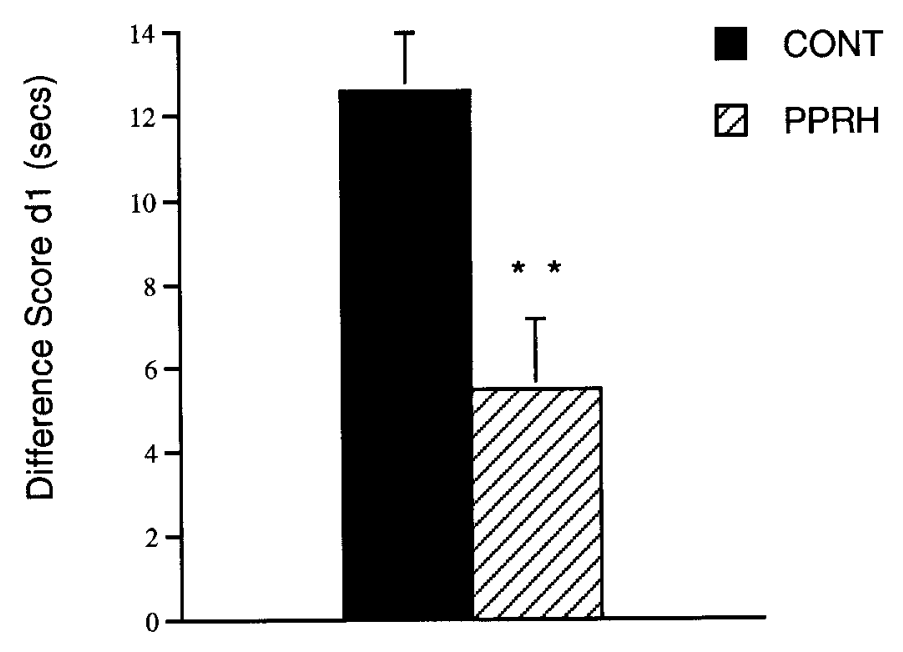

b

\section{Object Recognition}

d2

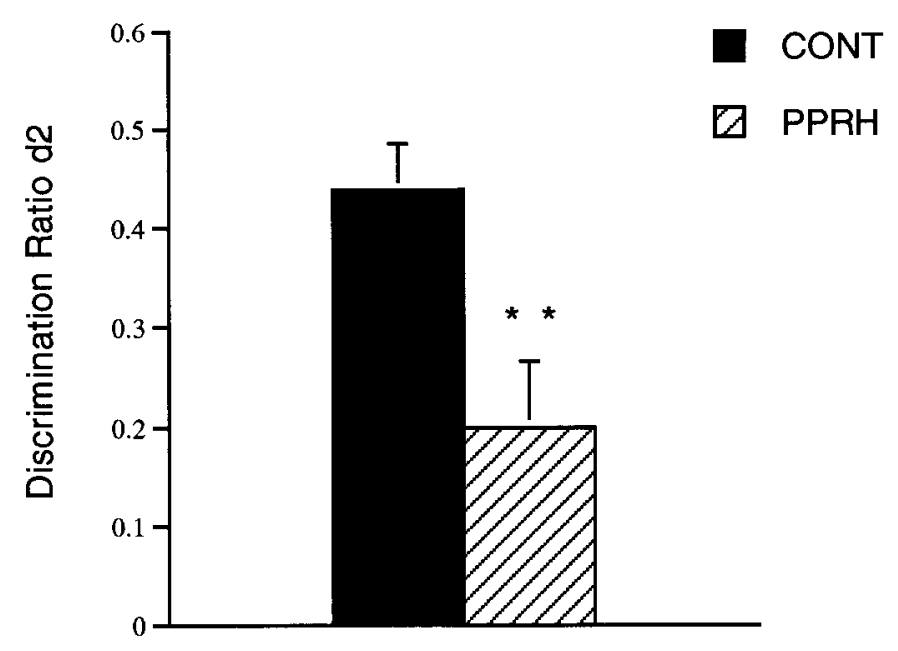

Figure 5. Performance of PPRH and CONT animals on the spontaneous recognition test. $a$, Difference in time spent exploring the novel and familiar objects $(d 1)$. $b$, Ratio of time spent exploring the novel object $(d 2) .{ }^{* *} p<0.01$, significantly poorer performance of PPRH animals relative to controls.

pocampus or fornix (Ennaceur et al., 1996, 1997; Murray and Mishkin, 1998) but instead depends on the perirhinal cortex (Meunier et al., 1993; Ennaceur et al., 1996). These results show that the perirhinal cortex and hippocampus can operate independently from one another, in a manner not predicted by current influential models (Squire and Zola-Morgan, 1991; Eichenbaum et al., 1994).

This is not the first study to indicate that perirhinal cortex lesions can spare spatial memory (Ennaceur et al., 1996; Aggleton et al., 1997; Ennaceur and Aggleton, 1997), but previous studies failed to include the postrhinal cortex and/or failed to use tasks that are unambiguously allocentric. The present findings show that the hippocampus does not require visual information from either the perirhinal or postrhinal cortices to mediate the performance of allocentric spatial tasks. This inevitably raises the question of how spatial information reaches the hippocampus. One possible clue comes from studies reporting that electrolytic lesions of perirhinal cortex can disrupt performance of spatial tasks in the water maze and radial arm maze (Wiig and Bilkey, 1994; Liu and Bilkey, 1998a,b). Comparison with the present study using axon-sparing excitotoxic lesions implicates fibers coursing through or adjacent to the perirhinal and postrhinal cortices. These fibers now require identification.

Whereas PPRH lesions had no effect on tests of spatial memory, these same lesions significantly disrupted spontaneous object recognition, thus confirming the efficacy of the lesions and replicating previous studies (Ennaceur et al., 1996; Aggleton et al., 1997; Ennaceur and Aggleton, 1997). Importantly, these lesions left intact the acquisition of simple object discriminations (Aggleton et al., 1997), showing that the object recognition deficit was not attributable to a general inability to discriminate objects. Furthermore, this sparing of object discrimination learning shows that the PPRH cortex is not necessary for all forms of visual discrimination. This is in agreement with recent reports showing that monkeys with lesions of perirhinal cortex are impaired on object discrimination only under certain circumstances (e.g., when the stimulus set size is large; Buckley and Gaffan, 1997). These results and the discrimination reversal deficit reported in the present study also show that deficits after perirhinal cortex lesions are not limited to recognition paradigms, consistent with the view that this region may have a more general role in "object identification” (Buckley and Gaffan, 1998; Murray et al., 1998).

In summary, the present dissociation not only shows that the perirhinal and postrhinal cortices are not necessary routes for visual information reaching the hippocampus but also that object familiarity-novelty detection depends on different neural substrates than do other aspects of event memory. This pattern of results cannot be accommodated within the notion of a unitary medial temporal lobe memory system and reveals the existence of important dissociations between different aspects of memory within the temporal lobe. Combined with other studies, these data support a dissociation between visual object processing in temporal cortex and spatial processing in the hippocampal system. At the same time, it must be acknowledged that these two forms of information will often be integrated so that specific objects can be situated within their spatial context. It is this integration for which connections between temporal cortex and hippocampus are most likely required. Indeed, in most situations, the encoding of complex event memories will require such integration. Thus, these regions are typically not independent of one another; rather, there is a continuum of situations along which they interact to varying degrees. The present study investigates phenomena at both ends of this continuum.

\section{REFERENCES}

Aggleton JP, Keen S, Warburton EC, Bussey TJ (1997) Extensive cytotoxic lesions involving both the rhinal cortices and area TE impair recognition but spare spatial alternation in the rat. Brain Res Bull 43:279-287.

Brown MW (1990) Why does the cortex have a hippocampus? In: Learn- 
ing and computational neuroscience: foundations of adaptive networks (Gabriel M, Moore J, eds). New York: MIT.

Buckley MJ, Gaffan D (1997) Impairment of visual objectdiscrimination learning after perirhinal cortex ablation. Behav Neurosci 111:467-475.

Buckley MJ, Gaffan D (1998) Perirhinal cortex ablation impairs visual object identification. J Neurosci 18:2268-2275.

Burwell RD, Witter MP, Amaral DG (1995) Perirhinal and postrhinal cortices of the rat: a review of the neuroanatomical literature and comparison with findings from the monkey brain. Hippocampus 5:390-408.

Bussey TJ, Muir JL, Everitt BJ, Robbins TW (1997) Triple dissociation of anterior cingulate, posterior cingulate, and medial frontal cortices on visual discrimination tasks using a touchscreen testing procedure in the rat. Behav Neurosci 111:920-936.

Deacon TW, Eichenbaum H, Rosenberg P, Eckmann KE (1983) Afferent connections of the perirhinal cortex in the rat. J Comp Neurol 220:168-190.

Dias R, Robbins TW, Roberts AC (1996) Primate analogue of the Wisconsin card sort test: effects of excitotoxic lesions of the prefrontal cortex in the marmoset. Behav Neurosci 110:872-886.

Dix SL, Aggleton JP (1998) Extending the spontaneous preference test of recognition: evidence of object-location and object-context recognition. Behav Brain Res, in press.

Eichenbaum H, Otto T, Cohen NJ (1994) Two functional components of the hippocampal memory system. Behav Brain Sci 17:449-518.

Ennaceur A, Aggleton JP (1997) The effects of neurotoxic lesions of the perirhinal cortex combined to fornix transection on object recognition memory in the rat. Behav Brain Res 88:181-193.

Ennaceur A, Neave N, Aggleton JP (1996) Neurotoxic lesions of the perirhinal cortex do not mimic the behavioural effects of fornix transection in the rat. Behav Brain Res 80:9-25.

Ennaceur A, Neave N, Aggleton JP (1997) Spontaneous object recognition and object location memory in rats: the effects of lesions in the cingulate cortices, the medial prefrontal cortex, the cingulum bundle and the fornix. Exp Brain Res 113:509-513.

Gaffan D (1994a) Dissociated effects of perirhinal cortex ablation, fornix transection and amygdalectomy: evidence for multiple memory systems in the primate temporal lobe. Exp Brain Res 99:411-422.

Gaffan D (1994b) Scene-specific memory for objects: a model of episodic memory impairment in monkeys with fornix transection. J Cognit Neurosci 6:305-320.

Gaffan D, Parker A (1996) Interaction of perirhinal cortex with the fornix-fimbria: memory for objects and "object-in-place" memory. J Neurosci 16:5864-5869.

Jones B, Mishkin M (1972) Limbic lesions and the problem of stimulusreinforcement associations. Exp Neurol 36:362-377.

Liu P, Bilkey DK (1998a) Lesions of perirhinal cortex produce spatial memory deficits in the radial maze. Hippocampus 8:114-121.

Liu P, Bilkey DK (1998b) Perirhinal cortex contributions to performance in the Morris water maze. Behav Neurosci 112:304-315.

Meunier M, Bachevalier J, Mishkin M, Murray EA (1993) Effects on visual recognition of combined and separate ablations of the entorhinal and perirhinal cortex in rhesus monkeys. J Neurosci 13:5418-5432.

Murray EA, Mishkin M (1998) Object recognition and location memory in monkeys with excitotoxic lesions of the amygdala and hippocampus. J Neurosci 18:6568-6582.

Murray EA, Málková L, Goulet S (1998) Crossmodal associations, intramodal associations, and object identification in macaque monkeys. In: Comparative neuropsychology (Milner AD, ed), pp 51-67. New York: Oxford UP.

Nadel L, Willner J (1980) Context and conditioning: a place for space. Physiol Psychol 8:218-228.

O'Keefe J, Nadel L (1978) The hippocampus as a cognitive map. New York: Oxford UP.

Paxinos G, Watson C (1997) The rat brain in stereotaxic coordinates. New York: Academic.

Squire LR, Zola-Morgan S (1991) The medial temporal lobe memory system. Science 253:1380-1386.

Suzuki W, Amaral DG (1994) Perirhinal and parahippocampal cortices of the macaque monkey: cortical afferents. J Comp Neurol 350: 497-533.

Wiig KA, Bilkey DK (1994) The effects of perirhinal cortical lesions on spatial reference memory in the rat. Behav Brain Res 63:101-109. 